Robert P. Panckow, Christopher McHardy, Alexander Rudolph, Michael Muthig, Jordanka Kostova, Mirco Wegener, Cornelia Rauh

\title{
Characterization of fast-growing foams in bottling processes by endoscopic imaging and convolutional neural networks
}

Journal article | Accepted manuscript (Postprint)

This version is available at https://doi.org/10.14279/depositonce-10267

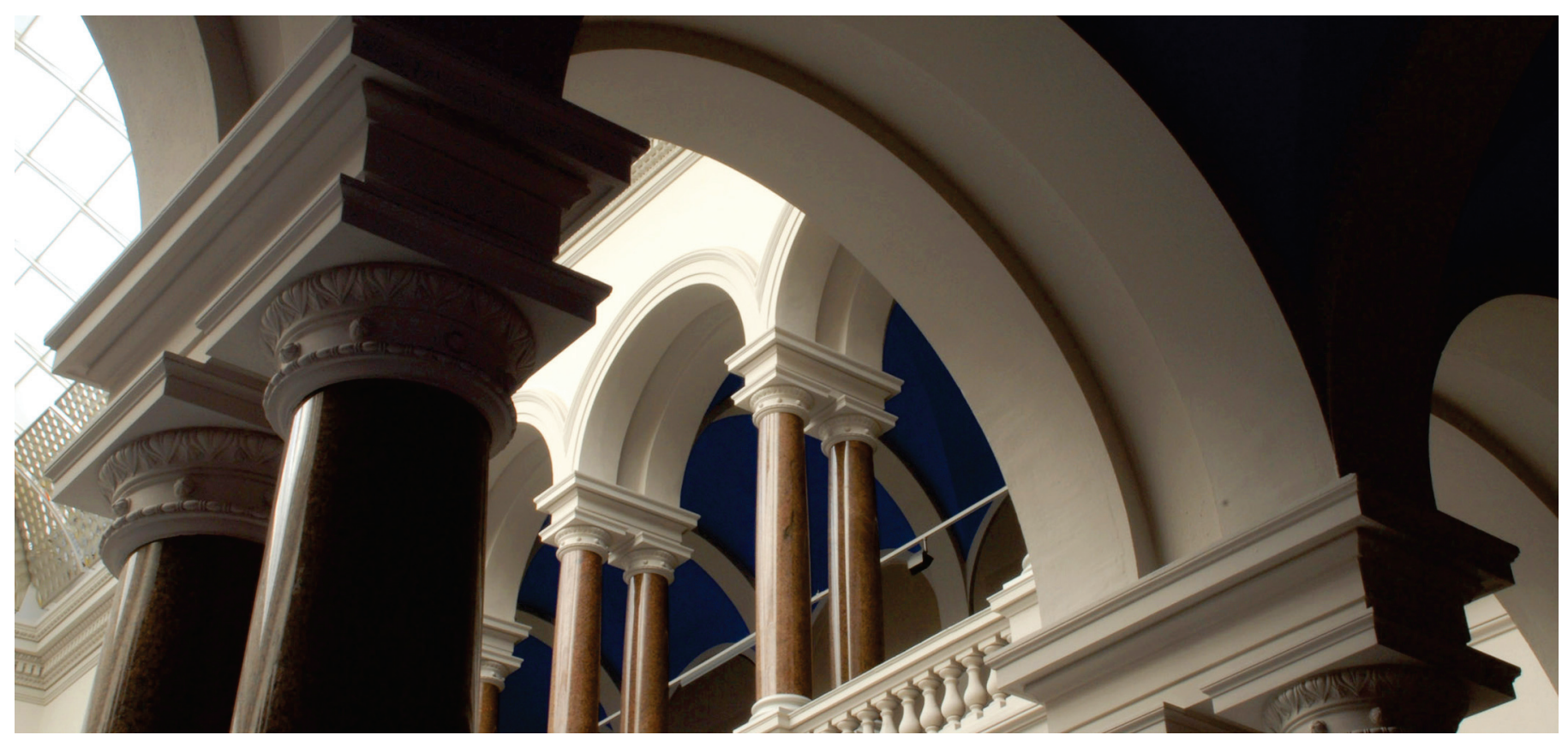

Panckow, R. P., McHardy, C., Rudolph, A., Muthig, M., Kostova, J., Wegener, M., \& Rauh, C. (2020).

Characterization of fast-growing foams in bottling processes by endoscopic imaging and convolutional

neural networks. Journal of Food Engineering, 110151. https://doi.org/10.1016/j.jfoodeng.2020.110151 


\title{
Characterization of fast-growing foams in bottling processes by endoscopic imaging and convolutional neural networks
}

\author{
Robert P. Panckow ${ }^{\mathrm{a}, \mathrm{c},}$, Christopher McHardy ${ }^{\mathrm{b}}$, Alexander Rudolph ${ }^{\mathrm{b}}$, Michael Muthig ${ }^{\mathrm{c}}$, Jordanka \\ Kostova $^{\mathrm{b}}$, Mirco Wegener ${ }^{\mathrm{c}}$, Cornelia Rauh ${ }^{\mathrm{b}}$ \\ a Technische Universität Berlin, Department of Chemical and Process Engineering, Fraunhoferstr. 33-36, 10587 Berlin, Germany \\ b Technische Universität Berlin, Department of Food Biotechnology and Food Process Engineering, Königin-Luise-Str. 22, 14195 Berlin, Germany \\ c SOPAT GmbH, Ordensmeisterstraße 15, 12099 Berlin, Germany
}

\begin{abstract}
Regardless of whether the occurrence of foams in industrial processes is desirable or not, the knowledge about the characteristics of their formation and morphology is crucial. This study addresses the measuring of characteristics in foam and the trailing bubbly liquid that result from air bubble entrainment by a plunging jet in the environment of industry-like bottling processes of noncarbonated beverages. Typically encountered during the bottling of fruit juices, this process configuration is characterized by very fast filling speeds with high dynamic system parameter changes. Especially in multiphase systems with a sensitive disperse phase like gas bubbles, the task of its measurement turns out to be difficult. The aim of the study is to develop and employ an image processing capability in real geometries under realistic industrial conditions, e.g. as opposed to a narrow measurement chamber. Therefore, a typically sized test bottle was only slightly modified to adapt an endoscopic measurement technique and to acquire image data in a minimally invasive way. Two convolutional neural networks (CNNs) were employed to analyze irregular non-overlapping bubbles and circular overlapping bubbles. CNNs provide a robust object recognition for varying image qualities and therefore can cover a broad range of process conditions at the cost of a time-consuming training process. The obtained single bubble and population measurements allow approximation, correlation and interpretation of the bubble size and shape distributions within the foam and in the bubbly liquid. The classification of the measured foam morphologies and the influence of operating conditions are presented. The applicability to the described test case as an industrial multiphase process reveals high potential for a huge field of operations for particle size and shape measurement by the introduced method.
\end{abstract}

\section{Keywords}

Plunging jet

Filling of beverages

Process monitoring

Image Analysis

Particle measurement

Bubble size distribution

\section{Highlights}

- Minimally invasive observation of foam and trailing bubbly liquid by endoscopes

- Measurement method in real geometries under industrial conditions is developed

- Robust object recognition for varying image qualities and operating conditions

- Convolutional neural networks need a time-consuming training process

- Broad applicability for measuring particle sizes and shapes in food processing

\footnotetext{
${ }^{*}$ Corresponding author.

E-mail addresses: panckow@tu-berlin.de (R.P. Panckow), christopher.mchardy@tu-berlin.de (C. McHardy); a.rudolph@tu-berlin.de (A. Rudolph); michael.muthig@sopat.de (M. Muthig); j.kostova@tu-berlin.de (J. Kostova); mirco.wegener@sopat.de (M. Wegener); cornelia.rauh@tu-berlin.de (C. Rauh)
}

This is the Accepted Manuscript of: Panckow, R. P., McHardy, C., Rudolph, A., Muthig, M., Kostova, J., Wegener, M., \& Rauh, C. (2020). Characterization of fast-growing foams in bottling processes by endoscopic imaging and convolutional neural networks. Journal of Food Engineering, 110151. https://doi.org/10.1016/j.jfoodeng.2020.110151 


\section{Introduction}

Foams are important in many areas of food technology and processing. Foods like baked goods, beverages, dairy products and desserts contain or entirely consist of foam, of which structure highly affects the food texture (Bamforth, 2004; Minor et al., 2009; Stanley et al., 1996) and aroma release (Dold et al., 2011; Mack et al., 2011; Reglitz, 2016), what finally impacts mouthfeel and sensory perception. Therefore, the characteristics of foams become an important quality measure of foamed foods. On the other hand, undesired foaming is a ubiquitous phenomenon in many processes of food production and other industries. For example, foam evolution may occur in heat exchangers, distillation plants, stirring vessels, degassers, fermentation tanks and filling processes (Schramm \& Wassmuth, 1994). In all of these processes, foaming can affect the transport of mass, momentum and heat, as well as chemical or biochemical reactions, and often leads to higher costs due to a reduced process performance and an increasing demand for process energy (Chandran Suja et al., 2018).

In both cases of desired or undesired foams, the appearance of the foam is important because it determines the quality attributes and the potential to disturb processes, respectively. Related quantities of interest are the bubble size distribution (BSD) and the foam morphology, which can be characterized by the bubble shape, polydispersity and the number of neighboring bubbles (Drenckhan \& Hutzler, 2015). Thereby, foams always show a certain degree of inhomogeneity and properties, such as liquid content or bubble sizes and shapes vary in space and time due to liquid drainage, coarsening and eventually bubble coalescence (Drenckhan \& Hutzler, 2015; Soleymani et al., 2013). These phenomena occur on very different time scales and do not only affect but also depend on the BSD and foam morphology (Cantat et al., 2013). For example, drainage in wet foams is relatively fast, while coarsening and coalescence play a minor role due to the thick liquid barrier between individual bubbles. In contrast, if a foam gets drier over time, the morphology of the foam changes, bubbles get closer to each other and gas diffusion along the gradient of the Laplace pressure becomes more important, finally leading to coarser bubbles (Saint-Jalmes, 2006).

Optical monitoring and digital image processing are widely applied to characterize the BSD in bubbly flows (Colombet et al., 2015; Schäfer et al., 2019; Ziegenhein et al., 2016) or foams (Barik \& Roy, 2009; Den Engelsen et al., 2002; Feitosa et al., 2006; Magrabi et al., 1999; Rami-shojaei et al., 2009). While the major challenge in bubbly flows lies in the detection and separation of overlapping bubbles, bubbles in dense layers must be separated in the case of foam, which entails different problems for the segmentation of individual bubbles. Typically, techniques, such as watershed transform (Borcherding et al., 2008; Germain \& Aguilera, 2012) and granulometry (Rami-shojaei et al., 2009) are applied for this task. These techniques require the identification of markers (seed points) in order to label individual bubbles as a basis of the segmentation. However, in industrial applications, images often contain multiple consecutive layers of gas bubbles, which make the automatic selection of markers error-prone and lead to over-segmentation (Hanbury, 2003). Moreover, most image processing methods strongly rely on stable illumination, homogeneous object intensities and low fluctuations in contrast (e.g. diffuseness, reflections) to keep the image processing steps working. In foams, however, light is diffused and images often suffer from intensity gradients (Vera et al., 2001). Consequently, conventional image segmentation techniques are difficult to apply and additional image preprocessing steps are required, although the results can still be unsatisfactory.

In the present work, a different technique is developed, which is based on endoscopic imaging and the application of convolutional neural networks (CNNs). CNNs can handle a broad variety of object appearances and provide an innovative and a new way to identify and segment bubbles in foams with inhomogeneous intensities. Therefore, an adequate characterization of foam morphology becomes possible. By means of a supervised network training approach based on annotated images, the CNNs are able to "interpret" that abstract image information by recognizing interrelated object features and reliably detect the desired objects. As an example of application, the new technique is applied in a lab-scale environment to study the morphology of foams arising during the filling of non-carbonated beverages. 
Beverage filling is among the fastest processes in food production and takes times in the order of seconds. A typical configuration of an industrial filling process consists of a receiving container, e.g. a bottle, which is located underneath a nozzle. Due to hygienic reasons many products are bottled in such a way that no parts of the filling machine dip into the bottled product (Blüml \& Fischer, 2004). Therefore, a characteristic of bottling processes is the presence of a liquid jet, which continuously entrains air into the product (Kiger \& Duncan, 2012). This leads to foam formation if the product contains a sufficient amount of surfactants. Typically, liquid non-carbonated foods, such as fruit juices are rich of surfactants so that the saturation of ascending bubbles with surfactant molecules occurs more or less instantaneously (Delgado et al., 2014) and the amount of generated foam is not restricted by the residence time of entrained air bubbles in the foaming liquid. Due to the short duration of the filling process and the continuous change of the foam morphology, the dynamic quantitative measurement of numbers, sizes and shapes of bubbles in a sensitive and unstable disperse system like foam is a major challenge. Therefore, the characterization of foam must ideally occur directly during the process in order to obtain a realistic picture. For this purpose, an in-situ analysis method with high spatial and temporal resolution was realized by connecting an endoscopic probe to a test bottle and recording photo-optical visuals of the bubbles within the growing foam layer. Thereafter, the developed image processing algorithms are applied to characterize foams arising during the filling of different fruit juices under industry-like conditions and thereby the power of the new analysis technique is demonstrated.

\section{Material and Methods}

\subsection{Experimental Setup}

The bottling trials were performed in a filling test rig, which is depicted in Fig. 1. The test rig consists of two connected containers between which the product is circulated by an impeller pump. The filling process starts by switching the position of a three-way valve so that the liquid flows into a test bottle. An additional pneumatic control valve is used to maintain a constant volume flow during the filling process. The maximum volume flow rate in the gravity-driven system is $\dot{V}_{\max }=0.157 \mathrm{~L} / \mathrm{s}$. The filling nozzle is a pipe with diameter of $10 \mathrm{~mm}$ and no additional internals, being located $32.6 \mathrm{~cm}$ above the bottle base. The test bottle is made of borosilicate glass with an internal diameter of $74 \mathrm{~mm}$ and contains three GL32 threads to connect the sensor probe with measuring position at the middle junction, see Fig. 1 (c). Further details about the sensor are given in Section 2.3.1.

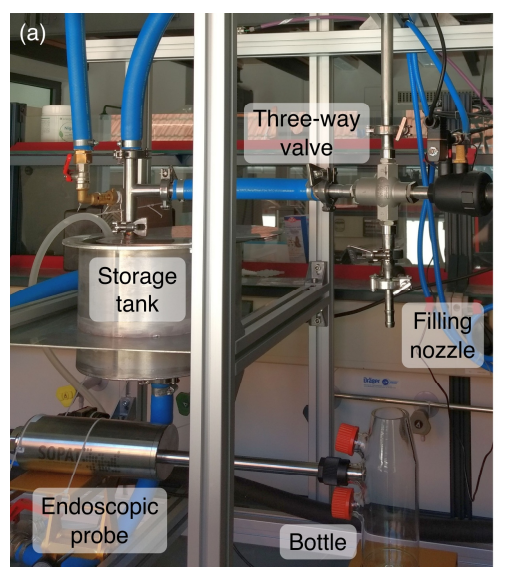

(b) 1: Storage tank

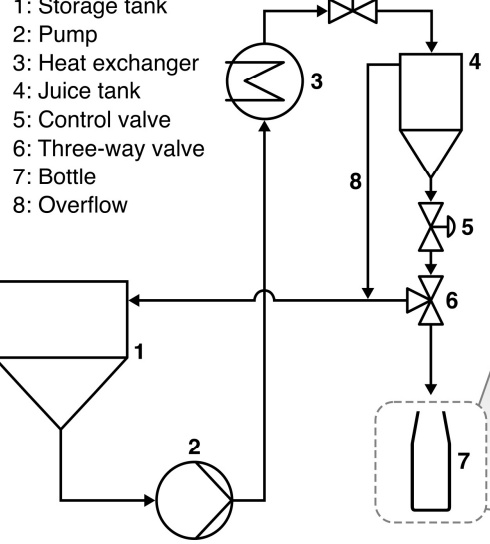

(c)

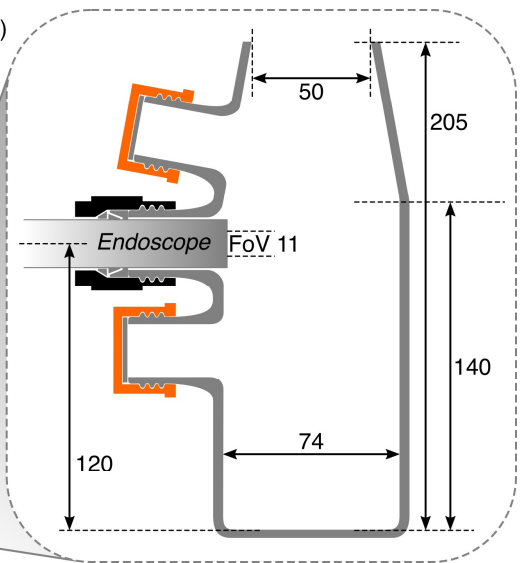

Fig. 1. Filling test rig used in the experiments: (a) photograph of the filling section with the optical measuring probe, (b) schematic drawing of the test stand with all components, (c) dimensions of the test bottle and probe's FoV with middle junction used as measuring position, all dimensions in $\mathrm{mm}$. 
The bottling trials with four different juices (see Section 2.2.1) were conducted at four different volume flow rates $(0.060 \ldots 0.157 \mathrm{~L} / \mathrm{s})$ at room temperature. The four different volume flow rates are qualitatively referred to as "Low", "LowMed", "MedHigh" and "High" due to minor deviations between the adjusted valve setpoints, see Tab. 1. Each experiment was repeated fivefold.

Tab. 1. Exact quantitative volume flows for each juice corresponding to the qualitative valve setpoints. Abbreviations for different juices are explained in Section 2.2.1.

\begin{tabular}{lcccc}
\hline \multirow{2}{*}{ Volume flow } & \multicolumn{4}{c}{$\dot{V} / \mathrm{L} \mathrm{s}^{-1}$} \\
\cline { 2 - 5 } & $\mathrm{PA}$ & $\mathrm{SC}$ & $\mathrm{MV}$ & $\mathrm{BR}$ \\
\hline Low & 0.071 & 0.060 & 0.071 & 0.073 \\
LowMed & 0.097 & 0.097 & 0.096 & 0.097 \\
MedHigh & 0.144 & 0.142 & 0.144 & 0.142 \\
High & 0.157 & 0.157 & 0.157 & 0.157 \\
\hline
\end{tabular}

\subsection{Material Characterization}

\subsubsection{Test Beverages}

The experiments were performed with four different commercial juices, namely Pineapple (PA), Sour Cherry (SC), Multivitamin (MV) and Beetroot (BR) juice. Physical properties of each juice were characterized as described in the following sections. The measured values are summarized in Tab. 2.

Tab. 2. Material properties of the different juices used in this study.

\begin{tabular}{cccccc}
\hline Juice & $\rho_{\mathrm{l}} / \mathrm{kg} \mathrm{m}^{-3}$ & $\eta_{\mathrm{l}} / \mathrm{Pa} \mathrm{s}$ & $k_{\mathrm{ow}} / \mathrm{Pa} \mathrm{s}^{n_{\text {ow }}}$ & $n_{\text {ow }} /-$ & $\sigma / \mathrm{mN} \mathrm{m}^{-1}$ \\
\hline $\mathrm{PA}$ & 1047.80 & - & 0.1286 & 0.421 & 43.5 \\
$\mathrm{SC}$ & 1057.88 & 0.0016 & - & - & 63.1 \\
$\mathrm{MV}$ & 1049.23 & - & 0.0109 & 0.761 & 31.2 \\
$\mathrm{BR}$ & 1041.84 & 0.0012 & - & - & 44.2 \\
\hline
\end{tabular}

\subsubsection{Density}

The mass density $\rho_{1}$ of all juices was measured with a density meter, which uses the oscillating u-tube technique (Anton Paar DMA $4100 \mathrm{M}$ ). The liquid temperature of $20^{\circ} \mathrm{C}$ was automatically controlled during the measurements. All measurements were conducted in triplicate.

\subsubsection{Dynamic Viscosity}

The dynamic viscosity $\eta_{1}$ of each juice was determined by means of a rheometer (Anton Paar MCR 302) with a double gap measuring system. After a pre-shearing stage, the shear rate was varied between $\dot{\gamma}=250 \mathrm{~s}^{-1}$ and $\dot{\gamma}=5 \mathrm{~s}^{-1}$ within $60 \mathrm{~s}$ in a linear ramp function. The dynamic viscosity of non-Newtonian beverages is described by Ostwald-de Waele model in Eq. (1).

$$
\eta_{\mathrm{l}}=k_{\mathrm{ow}} \dot{\gamma}^{n_{\mathrm{ow}}-1}
$$

Herein $k_{\mathrm{ow}}$ is the flow consistency index, $n_{\mathrm{ow}}$ is the flow behavior index. The temperature of $20^{\circ} \mathrm{C}$ was automatically controlled during the measurements. All experiments were conducted in triplicate. 


\subsubsection{Interfacial Tension}

The measurement of the interfacial tension $\sigma$ between air and the respective juices was carried out in a force tensiometer (Krüss K11) by means of Wilhelmy plate method. Prior to the measurement, all samples were pre-heated to the operating temperature of $20^{\circ} \mathrm{C}$. After an initial time until a stable value resulted, the interfacial tension was measured over a time interval of 5 minutes. All measurements were conducted in triplicate.

\subsection{Detection and Measurement of Bubbles}

\subsubsection{Raw Data Acquisition}

The photo-optical SOPAT measurement technique for particle sizing used in this study is capable of acquiring two-dimensional images of a dispersed phase as raw data (Maaß et al., 2012; Panckow et al., 2017). The measurement system consists of an endoscopic probe connected to a control unit and a workstation. On the latter, automated image analysis is performed to detect and measure the particles projected onto the camera sensor. In this study, the probe type SOPAT Kr was mounted in the middle GL32 thread, see Fig. 1 (c), to continuously monitor the passing, rising foam layer of bubbles during the running filling process. The endoscopic field of view (FoV) of $11 \mathrm{~mm}$, see Fig. 1 (c), covers the whole spectrum of occurring bubble sizes from two/three-digit microns up to the two-digit millimeter range, see scale in Fig. 2 (a). Each experiment consists of 250 images, which were continuously acquired during the filling process with a resolution of $2064 \times$ $1650 \mathrm{px}$ at a frame rate of $20 \mathrm{~Hz}$.
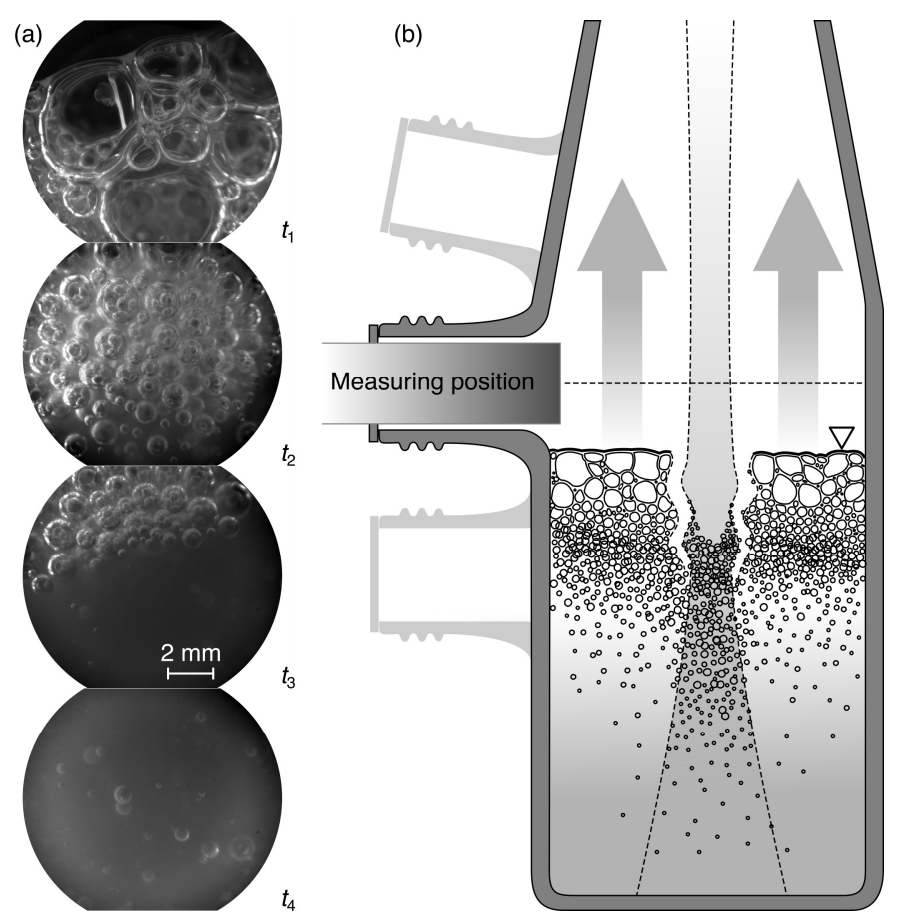

Fig. 2. Image data acquisition in the test bottle: (a) endoscopically acquired image material over time with $t_{1}<t_{2}<t_{3}<t_{4}$ (b) locally fixed, non-moving measuring position and rising foam layer with increasing time $t$.

As can be seen in Fig. 2 (a), a high diversity of image material results due to the rising level of the foam layer relative to the measurement position and different opacities of the investigated fruit juices. The endoscopically captured images within one single experiment are characterized by high overlapping with reflections, strong diffusiveness and extreme changes in contrast, constituting a formidable challenge to automated object recognition. 
The analysis of the acquired raw images and choice of algorithms were divided into two categories: detection of irregular bubbles in foam and detection of spherical bubbles in liquid, with their respective image processing described in the two subsequent sections. The transition was subjectively determined by visual observation and in Fig. 2 (a) corresponds to a division between times $t_{2}$ and $t_{3}$.

\subsubsection{Bubble Recognition in Foam Layer}

The consecutive image processing steps to analyze the first category of bubble appearances, namely irregular foam bubbles, are illustrated in Fig. 3.

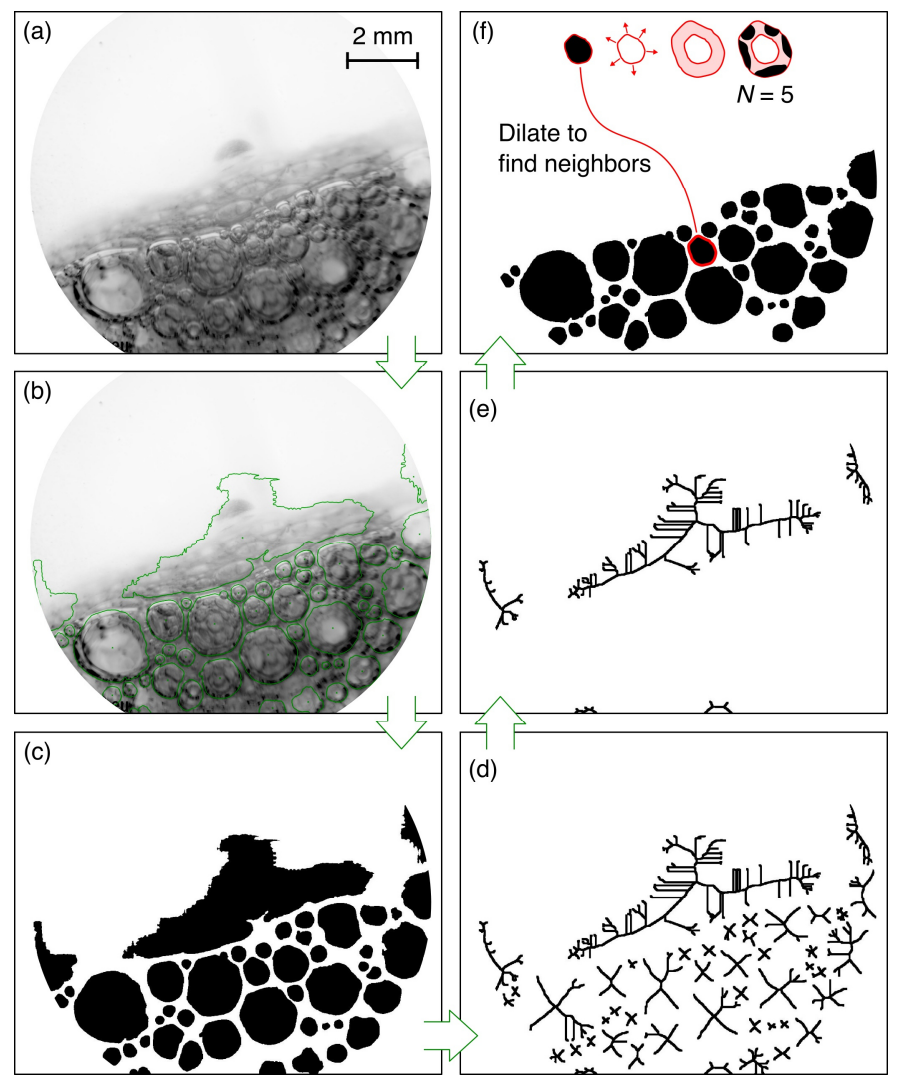

Fig. 3. Image processing chain from raw image to finally segmented objects: (a) endoscopic photograph, (b) output segmentation of applied $U$-Net, (c) binary of segmentation, (d) skeletonization, (e) branching threshold identifies detection errors, (f) binary segmentation after error removal. Quantities of foam morphology are measured in the final binary image (f), e.g. count of neighbors after dilation of each bubble. Images were inverted for better perceptibility.

The first step, applied to the raw images, see Fig. 3 (a), uses a $U$-net architecture (Ronneberger et al., 2015), a CNN implemented in the deep learning framework Caffe (Jia et al., 2014). It represents an end-to-end setting (raw image in, segmented image out), with its basic principle illustrated in Fig. 4: The network consists of two paths. Firstly, a contracting path (max pooling operations, see Fig. 4) is applied to the input image. In this path the layers are successively decreased in their $(x, y)$ dimensions, whereas the $z$ dimension increases. The contracting path is followed by an expansive path (up-convolutions, see Fig. 4) in which the layers get successively expanded in their $(x, y)$ dimensions and get smaller in the $z$ dimension. The final output layer consists of two segmentation matrices that are used to create a binary object segmentation mask, which indicates foreground objects and background.

The basic idea of this U-shaped network architecture is that the contracting path, by means of a sequence of convolutions, ReLU operations and max pooling operations, analyzes the information (e.g. objects) contained 


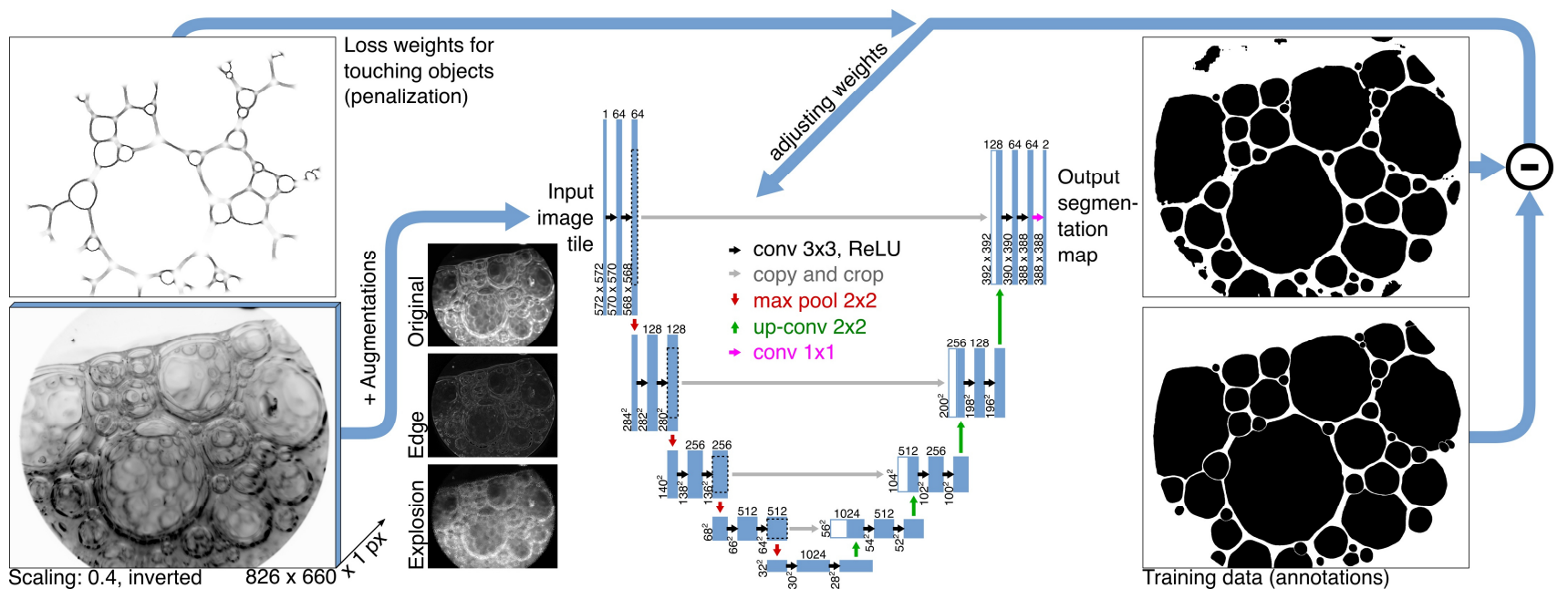

Fig. 4. Illustration of U-Net application with input/output, its architecture with contraction and expansion path, adapted with permission from Ronneberger et al. (2015), and the training process. The original image +3 augmentations (edge detection, explosion, inverting) in four different scalings $0.1,0.2,0.4,0.8$ were input. The prespecified annotations and pixel loss weights for touching objects (weight map) quantified the network weight adjustment.

in the image, increasing the „what" and decreasing the ,where“. The expansion path concatenates corresponding high-resolution features retrieved from this contracting path, see "copy and crop" in Fig. 4, and uses up-convolutions to synthesize a segmentation image by accurately allocating the object information to specific locations. Since the result is a segmentation image in which each pixel is assigned to the foreground (object) or background class and there is no further information to which object instance it belongs, this approach is incapable of detecting overlapping objects. However, irregular non-overlapping objects can be well detected. That implies, for this case of application, that the first layer of irregular shaped foam bubbles in front of the endoscopic probe is analyzed, compare Fig. 2.

By eroding close or touching objects and assigning loss weights for each pixel in the gap, a weight map defining additional penalization for accidental closure of gaps between objects is introduced, see Fig. 4. The capability of dealing with touching objects, makes the U-Net architecture particularly suitable for this case study with gas bubbles being divided by thin liquid films.

The neural net is trained in a supervised approach, meaning that in the training process a deviation from a desired output segmentation is used to adjust the network weights. The used training data consist of a number of images, which approximately covers the image and object variability within the experiment, plus associated, manually created annotations for each image, i.e., user-defined target segmentation masks. There were two training rounds with 15 images in the first round and 1 additional image in the second one. All 15 initial annotations have been revised in the second training round by increasing the contours' precision. For further augmentation of the training data, see Fig. 4, three extra manipulations (filters "Edge Detection", "Explosion" in the freeware tool IrfanView and inverting) as well as four different scalings $(0.1,0.2,0.4,0.8)$ were applied to each image, which furthermore expands the data pool by a factor of 16 . In the training process itself, the data are augmented by rotating, flipping, and slight distortions of the training data. This artificial data augmentation is important for two reasons: for reducing the effort of the tedious and time-consuming annotation process, and for increasing the net's ability to generalize. The network was then trained with the Adam solver of Caffe (Kingma \& Ba, 2017). Two criteria were examined during the training: the weighted "Cross Entropy Loss" (CEL) for all pixels should be minimized. The CEL function penalizes the deviation of a certain pixel classification from its predefined class (object or background) and is dependent on the introduced weight map, see Fig. 4 (Ronneberger et al., 2015). The second criterion "Intersection over Union" (IoU) should be maximized and is defined as the area of overlap between ground truth and prediction divided by their union. The training was assessed as "finished" when CEL and IoU reached a steady state (round 1: 500,000 Iterations, CEL=0.157, $\mathrm{IoU}=92.74 \%$; round 2: 857,500 Iterations, $\mathrm{CEL}=0.178, \quad \mathrm{IoU}=92.50 \%$ ). The computation took 
$0.499 \mathrm{~s} /$ Iteration on the measuring workstation, which is equipped with a six-core Intel ${ }^{\circledR}$ Xeon ${ }^{\circledR}$ CPU E5-1650 v4@3.60 GHz, 32 GB RAM and a NVIDIA TITAN X (Pascal ${ }^{\mathrm{TM}}$ architecture) with 12GB GDDR5X memory.

Although the output already shows outstanding segmentations for this highly complex image material, see

Fig. 3 (c), the U-Net was not trained for the upper (air phase ending) and lower boundaries (liquid bulk starting) of the foam layer. As can be seen in Fig. 3 (b) and (c), the resultant binary object segmentation images in those foam boundary regions show detection errors. Therefore, they were subsequently post-processed by a skeletonization, see Fig. 3 (d), in combination with an empirically quantified branching threshold, $\left(r_{i}<125\right) \wedge$ $\left(e_{i}<0.9\right)$ with $r_{i}$ being the ratio between the squared skeleton perimeter and the bubble area $A$, while $e_{i}$ is the eccentricity of a bubble $i$. Furthermore, bubbles touching the image border were removed to prevent a distortion of the measured quantities. After this postprocessing step, the final binary images allow measurement of the bubbles' number, sizes and shapes, see Fig. 3 (f). All postprocessing steps and quantity measurements were performed in the commercial software MATLAB ${ }^{\circledR}$ by MathWorks ${ }^{\circledR}$.

\subsubsection{Recognition of Trailing Bubbles}

The second analyzing method was employed to characterize the second category of bubble appearances, spherical bubbles in the trailing part after the main bulk of the foam layer passed by. It uses a homemade modification of the Faster R-CNN (Ren et al., 2017), such that the outputs are circle detections instead of bounding boxes. In the original work, the Faster $R$-CNN detects objects via bounding boxes in a two-step approach: firstly, a region proposal net proposes rectangular object candidates, which are subsequently fed to a detection net called Fast R-CNN (Girshick, 2015) that refines the candidate object locations and assigns them a label to an object class or background, i.e., the candidate is dismissed. The Faster $R-C N N$ achieves state-of-the-art object detection accuracy, while having good data throughput (ca. 0.4 full resolution images per second).

For augmentation of the training data, four kinds of flipping (without, horizontally, vertically, combined) and five different scalings $(0.900,0.925,0.950,0.975,1.000)$ were applied to each image in order to expand the data pool by a factor of 20. The network was then trained with the Stochastic Gradient Descent solver of Caffe (Bottou, 2012).

This implementation allows overlapping object detection but is limited to spherical shape detection. A big advantage in comparison to classic image analysis methods (Girshick et al., 2014) is the capability to deal with a broad variety of image material, i.e., varying lighting conditions and different appearances of bubbles in the images, with a single set of parametrization, see Fig. 5. A detected droplet, indicated by a green circle in the figure, is rated as true positive when its score, specified by a magenta number, exceeds a predefined threshold.

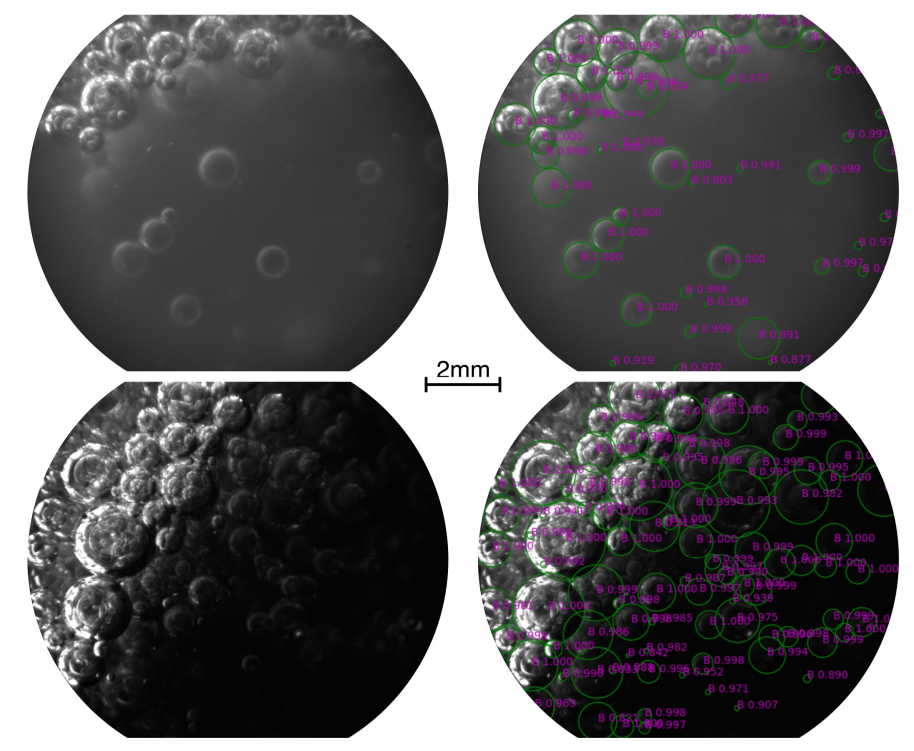

Fig. 5. Original images (left) and detections (right) in PA (top) and SC (bottom) juices. The overlapping spherical bubbles are detected by the implemented Faster R-CNN approach using a region proposal network, which was trained for different lighting conditions. The numerical output (magenta number) per object detection represents the score, i.e., the probability, for the existence of a particularly sized bubble at the respective position (green circle). 


\subsection{Foam and Process Characterization}

\subsubsection{Single Bubble Measurements}

Every single detected bubble was measured in size and shape. Based on the binary segmentation mask, see Fig. 3 (f), the area $A$ (total number of object pixels) and the perimeter $P$ (sum of its contour pixels) were obtained. As measure of size, the projected area diameter $d_{A}$ as equivalent diameter corresponding to the diameter of a sphere or circle with the same projected area as the bubble was calculated, according to Eq. (2).

$$
d_{A}=2 r_{A}=\sqrt{4 A / \pi}
$$

The index of sphericity $I_{\psi}$ (Ionescu-Tirgoviste et al., 2015) is given by Eq. (3) and describes how closely a bubble approaches a perfect sphere with $I_{\psi}=1$ or has a more elongated shape $I_{\psi} \rightarrow 2$.

$$
I_{\psi}=\frac{2}{\left(1+\pi d_{A} / P\right)}
$$

The number of neighboring bubbles $N$ is obtained after morphological square dilation (expansion) by an empirically determined width $(\approx 100 \mathrm{px})$ of every detected object and by counting the overlapped objects as illustrated in Fig. 3 (f).

\subsubsection{Bubble Population Measurements}

The holistic population properties of all $n$ bubbles define the foam morphology. Derived from the measured $d_{A}$ of each bubble, the arithmetic mean diameter $\left\langle d_{A}\right\rangle$ and the Sauter mean diameter $d_{32}$ of the ensemble are obtained following Eqs. (4) and (5), respectively.

$$
\begin{aligned}
\left\langle d_{A}\right\rangle & =\frac{1}{n} \sum_{i=1}^{n} d_{A i} \\
d_{32} & =\sum_{i=1}^{n} d_{A i}^{3} / \sum_{i=1}^{n} d_{A i}^{2}
\end{aligned}
$$

The average number of neighbors $\langle N\rangle$ and average index of sphericity $\left\langle I_{\psi}\right\rangle$ are analogously obtained to Eq. (4). To give a measure of the population's heterogeneity, the polydispersity $p_{32}$ (Drenckhan \& Hutzler, 2015) is calculated via Eq. (6).

$$
p_{32}=\frac{d_{32}}{2}\left(\frac{1}{n} \sum_{i=1}^{n} r_{A i}^{3}\right)^{-1 / 3}-1
$$

The measured BSDs are presented in their number-based cumulative form $Q_{0}$ and as box-and-whisker diagrams, with boxes ranging from $25^{\text {th }}$ to $75^{\text {th }}$ percentile, whiskers for \pm 1.5 interquartile range and visualizing outliers.

\subsubsection{Process Parameters}

By combining jet parameters in combination with material properties, an influence on the foam development can be described using the dimensionless Weber number We and the Capillary number Ca, see Eqs. (7) and (8), respectively (Kiger \& Duncan, 2012). 


$$
\begin{aligned}
\mathrm{We} & =\rho_{\mathrm{l}} u_{0}^{2} d_{0} / \sigma \\
\mathrm{Ca} & =u_{0} \eta_{\mathrm{l}} / \sigma
\end{aligned}
$$

Herein $u_{0}$ is the jet velocity at the nozzle outlet and $d_{0}$ is the initial jet diameter at the nozzle. In case of the two non-Newtonian juices PA and MV, the dynamic viscosity $\eta_{1}$ is replaced by the effective dynamic viscosity $\eta_{\text {eff }}$ (Safouane et al., 2006) with its apparent shear rate $\dot{\gamma}_{\mathrm{a}}$ (Senge et al., 2004), see Eq. (9),

$$
\eta_{\mathrm{eff}}=k_{\mathrm{ow}} \dot{\gamma}_{\mathrm{a}}^{n_{\mathrm{ow}}-1}=k_{\mathrm{ow}}\left(4 \dot{V} \pi^{-1} r_{0}^{-3}\right)^{n_{\mathrm{ow}}-1}
$$

where $r_{0}$ equals the radius of the nozzle outlet.

\section{Results}

\subsection{Determination of BSDs in Foams}

In order to test the developed CNN image processing, bubble and foam characteristics were determined from the recorded images. Therefore, different distribution functions are fitted to the measured diameters of the bubble populations for different juices and filling speeds. The goodness of fit $\phi$ was estimated as the mean absolute error (average residuals) with Eq. (10)

$$
\phi=\frac{1}{n_{\mathrm{bin}}} \sqrt{\sum_{i=1}^{n_{\mathrm{bin}}}\left(f\left(d_{A i}\right)-y_{i}\left(d_{A i}\right)\right)^{2}}
$$

where $f\left(d_{A i}\right)$ is the value of the respective continuous cumulative distribution function for a bubble diameter $d_{A i}$ and $y_{i}$ is the experimentally determined value of the discrete cumulative distribution, with $n_{\text {bin }}$ being the number of bins. Due to the presence of some very large bubbles at the foam-air interface, Freedman-Diaconis rule was applied for the binning of the individual bubbles, since it is suitable for heavily tailed data sets. The number of recorded bubbles for each juice and volume flow ranges between 1109 and 7347, depending on the foam height, filling speed and foam characteristics. On average, 4923.9 bubbles (standard deviation 1870.0) were evaluated for each operating point.

\begin{tabular}{|c|c|c|c|c|c|c|c|c|c|c|c|c|}
\hline \multirow{2}{*}{ Vol. flow } & \multicolumn{4}{|c|}{ Log-normal / $10^{-3}$} & \multicolumn{4}{|c|}{ Weibull / $10^{-3}$} & \multicolumn{4}{|c|}{ Gamma / $10^{-3}$} \\
\hline & $\mathrm{PA}$ & $\mathrm{SC}$ & MV & $B R$ & PA & SC & MV & $\mathrm{BR}$ & PA & SC & MV & $\mathrm{BR}$ \\
\hline Low & 1.9 & 3.4 & 1.0 & 0.6 & 3.9 & 2.2 & 2.6 & 1.9 & 3.6 & 1.9 & 1.8 & 1.3 \\
\hline LowMed & 1.2 & 0.7 & 0.9 & 0.4 & 0.7 & 0.9 & 1.7 & 1.2 & 0.6 & 0.4 & 1.1 & 0.8 \\
\hline MedHigh & 0.9 & 1.0 & 0.8 & 1.3 & 0.8 & 1.1 & 1.2 & 1.0 & 0.4 & 0.6 & 0.7 & 0.6 \\
\hline High & 0.9 & 1.0 & 1.5 & 0.8 & 0.8 & 1.4 & 1.2 & 0.8 & 0.4 & 0.8 & 0.6 & 0.4 \\
\hline Average & 1.2 & 1.5 & 1.0 & 0.8 & 1.6 & 1.4 & 1.7 & 1.2 & 1.2 & 1.0 & 0.9 & 0.8 \\
\hline Overall & \multicolumn{4}{|c|}{$1.14(0.69)$} & \multicolumn{4}{|c|}{$1.46(0.86)$} & \multicolumn{4}{|c|}{$0.99(0.84)$} \\
\hline
\end{tabular}

Tab. 3. Goodness of fit $\phi$ for different distribution functions for the description of the BSD. Numerical values quantify $\phi$ with respect to the qualitative filling speed (cf. Tab. 1), the average of all filling speeds and the overall average of the whole data set with standard deviation in brackets.

Tab. 3 compares the goodness of fit for the log-normal, Weibull and Gamma distributions, which were all previously applied to characterize the BSD in foams (Den Engelsen et al., 2002). Generally, all models describe the data fairly well and the average residuals are about three orders of magnitude smaller than the distribution itself. The worst fits are obtained at low filling speeds and this can be explained by the fact that the smallest amount of foam was generated under those conditions. If the filling speed increases, the bubble population can be fitted more precisely by any of the tested models. Among the different models, the lowest overall error is 
achieved by the Gamma distribution, although the variation of error within the samples is lowest for the lognormal distribution. In contrast, Weibull distribution is the worst in terms of both criteria, mainly due to an inaccurate description of the smallest bubbles. Based on these results and considering that only the parameters of the log-normal distribution imply physical meaning, it is recommended to apply this model to describe the BSD of foams which are created by a plunging jet. The corresponding parameters of the estimated BSDs are shown in Tab. 4. The logarithmic mean diameters $\mu_{\log }$ correspond to values between 400 and $700 \mu \mathrm{m}$, approximately linearly increasing with the filling speed.

Tab. 4. Parameters of the fitted log-normal distributions in foams of different juices with respect to the filling speed.

\begin{tabular}{|c|c|c|c|c|c|c|c|c|}
\hline \multirow[b]{2}{*}{ Vol. flow } & \multicolumn{4}{|c|}{ Mean of log. diameters $\mu_{\log }$} & \multicolumn{4}{|c|}{ Std. dev. of log. diameters $\sigma_{\log }$} \\
\hline & PA & SC & MV & $\mathrm{BR}$ & PA & SC & MV & $B R$ \\
\hline Low & -0.9410 & -0.6835 & -0.6322 & -0.7744 & 0.8376 & 0.6293 & 0.6604 & 0.6771 \\
\hline LowMed & -0.7424 & -0.6139 & -0.5019 & -0.6580 & 0.6678 & 0.5922 & 0.7102 & 0.6734 \\
\hline MedHigh & -0.4045 & -0.5484 & -0.4173 & -0.5041 & 0.6339 & 0.6511 & 0.6778 & 0.6579 \\
\hline High & -0.4680 & -0.5301 & -0.4001 & -0.5229 & 0.6373 & 0.6648 & 0.6713 & 0.6562 \\
\hline
\end{tabular}

Fig. 6 (a) shows the cumulative BSD in foams of SC juice at different filling speeds. The majority of bubbles in the foams have sizes $d_{A}$ approximately between 0.2 and $2 \mathrm{~mm}$ with few outliers, ranging up to $5 \mathrm{~mm}$, see Fig. 6 (b), ordinate axis capped at $3.5 \mathrm{~mm}$. It can be seen that the BSD shifts towards larger bubbles at higher filling speed, Fig. 6 (a), what is also reflected in the average bubble diameter ( $\mu_{\text {log }}$ increases, see Tab. 4). Similar trends were observed for the other bottled juices. An evaluation of the interquartile distance of the box plots in Fig. 6 (b) shows a broadening of the BSD for increasing filling speed. Likewise, the number and size of large bubbles increases.

The maximum size of the primary bubbles at the impact point of the plunging jet is controlled by the jet Weber number (Evans et al., 1992). However, the BSD in a foam under the investigated conditions can be potentially affected by additional phenomena. These include break-up and coalescence of dispersed gas bubbles in the liquid phase and the increase of the bubble size over time in the foam due to coarsening. All these phenomena are affected by the physical material properties, such as density, surface tension or dynamic viscosity, so that the jet velocity alone cannot provide a complete description of the bubble size in foams.
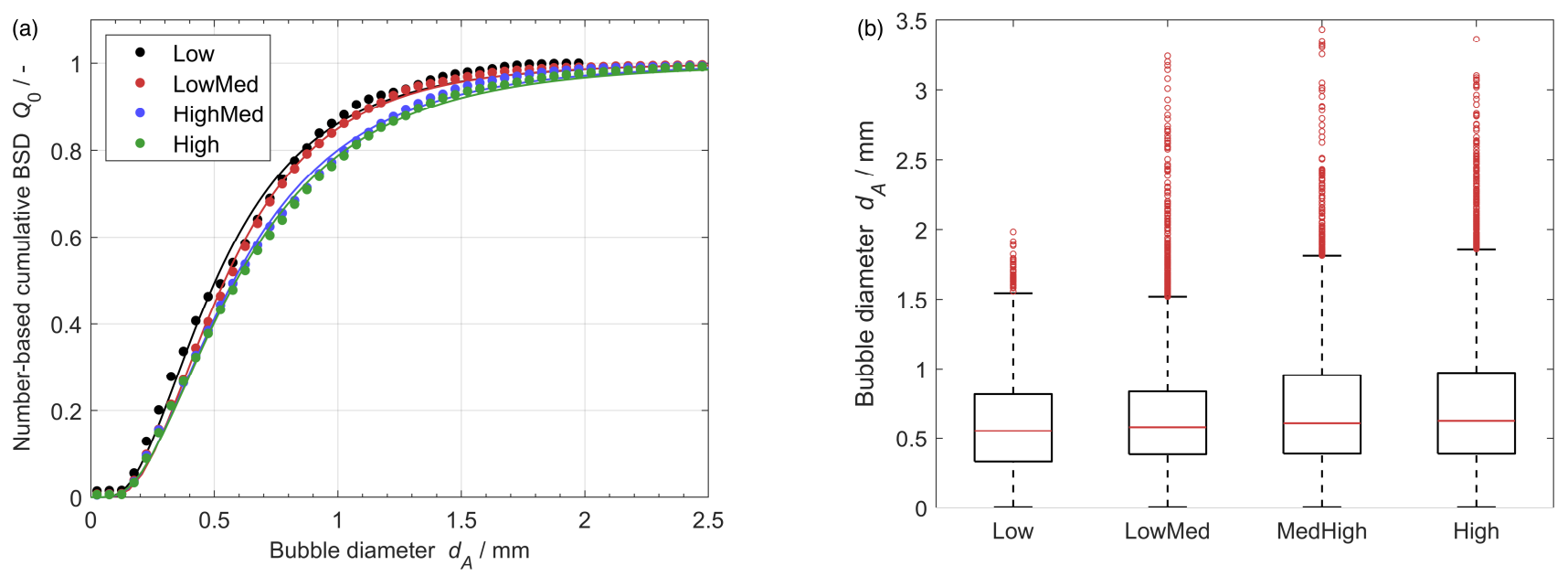

Fig. 6. (a) Measured (symbols) and fitted (lines, log-normal model) BSDs in foams of SC juice resulting from different filling speeds. (b) Box plots of the same bubble populations. 
Dimensionless numbers, such as We and Ca relate inertial, viscous and interfacial forces and are known to control the gas entrainment by plunging liquid jets (Drenckhan \& Saint-Jalmes, 2015; Kiger \& Duncan, 2012) and the mechanism of bubble generation (Bertola et al., 2018). As an example, Fig. 7 depicts the $10^{\text {th }}, 50^{\text {th }}$ and $90^{\text {th }}$ percentiles of the number-based BSD $\left(d_{\mathrm{n} 10}, d_{\mathrm{n} 50}\right.$ and $d_{\mathrm{n} 90}$ respectively) with respect to Ca. Lower values of Ca correspond to smaller bubbles, which reflects the effects of a higher interfacial tension $\sigma$ on the bubble size. On the other hand, one finds that higher viscous forces on the jet correspond to a slightly larger bubble diameter $d_{A}$ in the foam. This result is quite in agreement with the experiments of (Bertola et al., 2018), who observed that larger primary bubbles are formed if the jet velocity increases, thus at higher values of $\mathrm{Ca}$. In the present work, the observed relations between $\mathrm{Ca}$ and the percentiles of the BSD are approximately linear. Thereby, it should be noted that two of the four juices (PA, MV) were found to be non-Newtonian fluids and an apparent shear rate $\dot{\gamma}_{\mathrm{a}}$ was assumed for the evaluation of the dynamic viscosity, see Eq. (9). The results for PA juice do not entirely confirm the trends found for the other three tested beverages, while congruence with the general trends is only given at higher volume flows. This discrepancy might be caused by errors of planimetric measurement in heterogeneous foams (Cheng \& Lemlich, 1983) or sampling errors due to the small amounts of foam formed at low filling speeds. Furthermore, the calculation of a representative dynamic viscosity (here approximated by $\eta_{\text {eff }}$ ) of a non-Newtonian liquid turned out to be difficult due to the unknown shear rate in the impact zone of a plunging jet (Politova et al., 2018). The developed CNN proves a suitable tool for detailed investigations on the relations between process effects, material characteristics and the resulting foam properties. Based on the presented measuring tools and methods, future research under process conditions will expand the data pool of the exemplarily shown results.

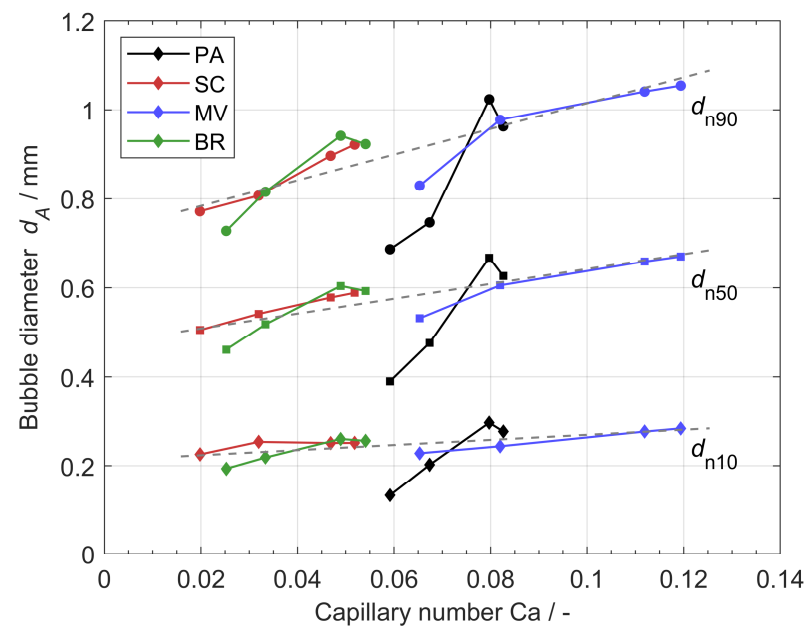

Fig. 7. Percentiles of the BSD in foams for different juices with respect to the Capillary number. Colors encode different juices and symbols different percentiles of the BSD. The dashed lines indicate linear regressions for which pineapple juice (PA) was not considered.

\subsection{Characterization of Foam Morphology}

Beside the BSD, several other parameters determine the morphology of foams, which can also be assessed by automatic image processing based on the developed $\mathrm{CNN}$ approach. Among these, polydispersity $p_{32}$, index of sphericity $I_{\psi}$ and the number of neighbors $N$ of each bubble are important quantities (Drenckhan \& Hutzler, 2015). Tab. 5 presents those quantities, which result from different juices at different filling speeds. As can be seen, all quantities are mainly unaffected by the filling conditions and materials, so that the overall average values are further regarded.

The detected average number of neighbors $\langle N\rangle$ in the two-dimensional visuals is approximately equal to 4 (3.9713). It can be assumed that this value corresponds to a number of 6 neighbors in three-dimensional foams (van Hecke, 2010). For disordered foams, this configuration is typical for the jamming point (Drenckhan \& Hutzler, 2015), which characterizes the transition of a bubbly liquid to a foam. 
Tab. 5. Average number of neighbors, average sphericity and polydispersity in foams of different juices with respect to the filling speed.

\begin{tabular}{|c|c|c|c|c|c|c|c|c|c|c|c|c|}
\hline \multirow[b]{2}{*}{ Vol. flow } & \multicolumn{4}{|c|}{ Average neighbors $\langle N\rangle$} & \multicolumn{4}{|c|}{ Average sphericity $\left\langle I_{\psi}\right\rangle$} & \multicolumn{4}{|c|}{ Polydispersity $p_{32}$} \\
\hline & PA & SC & MV & $B R$ & PA & SC & MV & $B R$ & PA & SC & MV & $B R$ \\
\hline Low & 2.286 & 3.354 & 3.821 & 4.440 & 1.042 & 1.031 & 1.018 & 1.020 & 0.607 & 0.260 & 0.377 & 0.454 \\
\hline LowMed & 4.126 & 4.511 & 4.180 & 4.656 & 1.026 & 1.022 & 1.016 & 1.019 & 0.424 & 0.338 & 0.486 & 0.426 \\
\hline MedHigh & 3.887 & 4.222 & 3.889 & 4.538 & 1.019 & 1.023 & 1.018 & 1.021 & 0.387 & 0.408 & 0.463 & 0.380 \\
\hline High & 3.544 & 4.065 & 3.788 & 4.233 & 1.016 & 1.024 & 1.016 & 1.022 & 0.444 & 0.371 & 0.422 & 0.371 \\
\hline Average & 3.461 & 4.038 & 3.920 & 4.466 & 1.026 & 1.025 & 1.017 & 1.020 & 0.466 & 0.344 & 0.438 & 0.408 \\
\hline Overall & \multicolumn{4}{|c|}{$3.9713(0.5762)$} & \multicolumn{4}{|c|}{$1.0221(0.0067)$} & \multicolumn{4}{|c|}{$0.4138(0.0752)$} \\
\hline
\end{tabular}

Therefore, the observed average number of neighboring bubbles in the foam indicates a disordered foam with high liquid content for all conditions. This is reasonable since the duration of the filling process is in the order of seconds so that just a fraction of the liquid phase might have been drained out of the foam.

Typically, bubbles in wet foams are spherical, proven by the average index of sphericity $\left\langle I_{\psi}\right\rangle$. According to Eq. (3), a value of 1 corresponds to a perfect sphere. The measured values of $\left\langle I_{\psi}\right\rangle$ are found to be between 1.016 and 1.042 in all foams. For the entirety of all tested conditions, the average value is found to be 1.022 , which means that mainly spherical bubbles are present in the generated foams.

Another parameter of interest is the polydispersity $p_{32}$ of the bubble population, see Eq. (6). The measured values of the polydispersity for the different foams range between 0.260 and 0.607 , while the global average of all foams is 0.4138 . According to Drenckhan and Hutzler (2015), typical values for the polydispersity are below 0.5 , whereby monodisperse foams can be assumed for $p_{32}<0.05$. Therefore, the measurements indicate highly polydisperse foams under all conditions. It should be noted that extreme values of all criteria were observed under low filling speed conditions, which might be due to measurement uncertainties, as mentioned in the previous section.

\subsection{Characterization of Bubbly Flow}

As soon as the foam has passed the sensor probe, trailing bubbles are observed, see Fig. 2 (a), which can be characterized by the implemented Faster R-CNN modification, described in Section 2.3.3. The Sauter mean diameter $d_{32}$ and the arithmetic mean diameter $\left\langle d_{A}\right\rangle$ of the dispersed bubbles are presented in Tab. 6. The observed gas bubbles were spherical for all investigated conditions.

Tab. 6. Comparison of two diameter averages for trailing bubble populations in different juices with respect to the filling speed.

\begin{tabular}{|c|c|c|c|c|c|c|c|c|}
\hline \multirow[b]{2}{*}{ Vol. flow } & \multicolumn{4}{|c|}{ Sauter mean diameter $d_{32} / \mathrm{mm}$} & \multicolumn{4}{|c|}{ Arithmetic mean diameter $\left\langle d_{A}\right\rangle / \mathrm{mm}$} \\
\hline & PA & SC & MV & $\mathrm{BR}$ & PA & SC & MV & BR \\
\hline Low & 0.225 & 0.193 & 0.351 & 0.518 & 0.123 & 0.127 & 0.141 & 0.132 \\
\hline LowMed & 0.390 & 0.642 & 0.647 & 0.705 & 0.143 & 0.192 & 0.251 & 0.180 \\
\hline MedHigh & 0.906 & 1.006 & 0.965 & 1.049 & 0.222 & 0.442 & 0.391 & 0.456 \\
\hline High & 0.912 & 1.031 & 0.999 & 1.073 & 0.307 & 0.537 & 0.407 & 0.527 \\
\hline
\end{tabular}

A non-dimensional representation of the bubble size is given by the Laplace number $\mathrm{La}=\left\langle d_{A}\right\rangle \rho_{1} \sigma / \eta_{1}^{2}$, which is related to the stability or rupture of gas ligaments at the jet impact point (Drenckhan \& Saint-Jalmes, 2015). It can also be expected that the average bubble size in the liquid is related to the Weber number We, which relates the inertial forces on gas ligaments to the stabilizing interfacial forces, see Eq. (7). Based on the measured sizes of trailing bubbles, linear relationships $\mathrm{La}=c_{1} \mathrm{We}+c_{2}$ were found for the Newtonian (SC, BR, $\left.\mathrm{R}^{2}=0.986\right)$ and for the non-Newtonian juices (PA, MV, $\left.\mathrm{R}^{2}=0.911\right)$. In both cases, the respective intercept $c_{2}$ is about one order of magnitude smaller than the smallest value of La, so that the regression line approximately passes through the origin. By neglecting the intercept and by solving for $\left\langle d_{A}\right\rangle$ a quadratic dependency 
on the Capillary number $\left\langle d_{A}\right\rangle \approx c_{1} d_{0} \mathrm{Ca}^{2}$ is obtained. The definition of the apparent viscosity according to Eq. (9) provides a possible explanation for the fact that no coinciding regression was obtained for Newtonian and non-Newtonian juices, since the apparent shear rate $\dot{\gamma}_{\mathrm{a}}$ represents the conditions in the nozzle rather than at the jet impact point. Although it is possible to approximate $\dot{\gamma}_{\mathrm{a}}$ by means of an approximated jet velocity $u_{j} \approx$ $\left(u_{0}+2 g h(t)\right)^{0.5}$ and jet diameter $d_{j} \approx d_{0}\left(u_{0} / u_{j}\right)^{0.5}$ at the impact point (Roberts \& Rao, 2011), it must be considered that the height of fall of the jet $h(t)$ decreases during the filling process and, consequently, the quantities $u_{j}$ and $d_{j}$ depend on time. Thus, the approximated apparent shear rate $\dot{\gamma}_{\mathrm{a}}$ as well as the viscosity of non-Newtonian juices at the jet impact point are not constant, which is why the approximation is not appropriate to be applied in the context of a high-speed filling process.

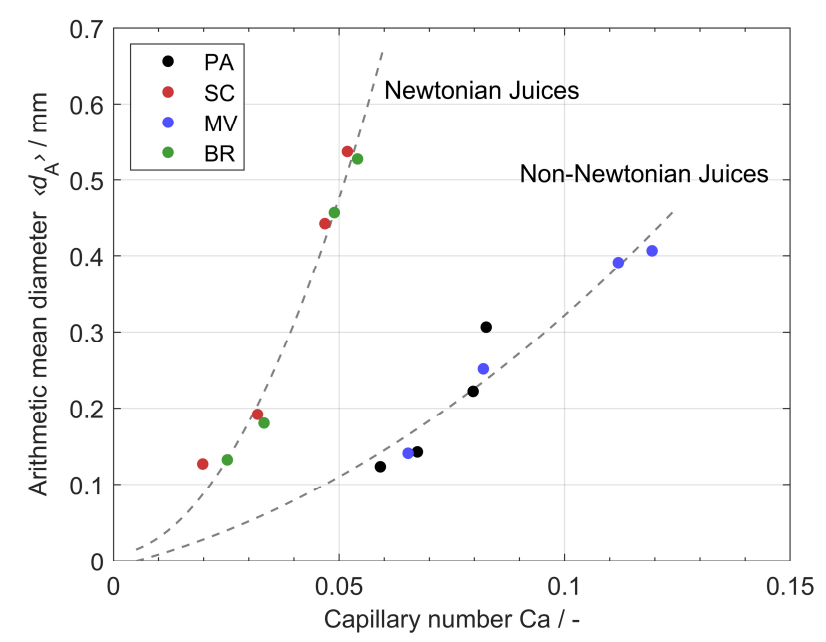

Fig. 8. Arithmetic mean diameter for different juices and filling conditions with respect to the Capillary number. Regression lines indicate the derived quadratic approximation.

Fig. 8 shows the arithmetic mean diameter $\left\langle d_{A}\right\rangle$ of the dispersed bubbles with respect to Ca as defined by Eq. (8). The derived quadratic approximation is indicated by the regression lines. On average, 10,144 bubbles were evaluated per data point (min: 4350, max: 25,130). It can be seen that larger bubbles are formed if Ca increases, but also that the average bubble size is generally smaller than in foam, see also Fig. 7. Thereby, no clear correlation between bubble sizes in the bubbly liquid and in the foam was found, although a weak trend indicated that bubbles in the foam were larger if the entrained bubbles were larger as well. Generally, the filling speed has a significant impact on the bubble size in the trailing flow, while the quantiles of the BSD in foam are very similar at different filling conditions. A reason for that might be that even though the primary bubbles are smaller at low filling speeds, their residence time in the foam increases at such conditions. Therefore, the BSD might be affected by additional mechanisms, such as coarsening over time (Saint-Jalmes, 2006). The measured data indicate that such mechanisms are important even during the relative short duration of the bottling process. However, the presented data do not allow to investigate the occurrence of coarsening or other mechanisms in detail, but they show the potential of the developed CNN for characterizing disperse systems and therefore, its feasibility for performing mechanistic studies in future research to obtain a complete picture. This would be a desirable task for the plunging jet configuration, since, to the best of the authors' knowledge, no clear understanding of determining factors for the BSD exists, specifically in case of non-Newtonian fluids representing many foodstuffs.

\section{Discussion}

\subsection{Using Neural Networks for Bubble Detection in Foams}

The implementation of the two described CNNs for recognition of close or touching objects with irregular shape as well as circular overlapping objects turned out to be a successful combination for image analysis in bubbly liquids, wet foams and their transition. 
However, since both CNNs are based on a supervised approach, they need a tremendous amount of predefined labeled image data. This problem is tackled by data augmentation to artificially expand the pool of training data for the networks. Still, annotating the image data acquired in a specific application remains a time-consuming, but necessary step to adapt the CNN usage to a concrete case study. Furthermore, it is of high importance to cover the whole spectrum of image appearances, which can possibly occur in a specific system. The annotations need to be very consistent, e.g. no sloppy annotation of particle contours and strict compliance with conventions like thresholds for sharpness or overlapping, which can be quite a challenge to judge subjectively during the annotation process.

Since the trailing bubbles are spherical and those in the foam are almost spherical, both bubble appearances could have been analyzed by only one image analysis approach, which is the implemented Faster R-CNN modification to detect spherical overlapping objects. However, two different $C N N$ techniques were chosen to best satisfy the demands on the image analysis for the respective task. In foam, lamella disturb the bubble detection and dense layers of irregular shaped bubbles are present, of which only the first layer in front of the endoscopic probe should be examined. In the bubbly flow, the challenge is to detect and separate superimposed bubbles with strong differences in image contrast. The aim was to achieve robustly and reliably working image analyses for those two specific tasks instead of aiming at a universal approach. In addition to that, the latter would have required a very high amount of newly annotated training data to interpret the non-spherical bubble shapes as circles within the foam, while keeping the same quality of detection for the trailing bubbles.

Considering those requirements on the difficult training process of the CNNs, they provide a very powerful image analysis tool with striking advantages in performance over conservative image processing chains, which are constrained to strict boundaries of variations in illumination, resulting image contrasts, limited particle shapes and high calculation times. As opposed to this, by not being bound to a specific setup with constrained lighting, e.g. in a measurement chamber or bypass, but freely operating in an arbitrary spatial setup and still handling challenges like different juice diffusivities/reflectivities open a huge range of applications. Beside the presented case study in four different juices and a limited amount of data points, the described image analysis in combination with an industrial-suited image acquisition system can be applied as process monitoring tool to control industrial applications where foaming occurs.

\subsection{Bubble Size Distributions and Foam Morphology}

The developed CNNs allow an accurate description of bubble sizes and shapes in images of foams and bubbly liquids. Generally, the generated data indicate that foams, which arise in bottling processes of noncarbonated beverages, are wet, disordered and polydisperse with an average bubble size in the order of $400 \ldots 700 \mu \mathrm{m}$. The investigated bottling process shows similarities to Ross-Miles foamability test, which also follows the basic principle that a test liquid plunges into a reservoir of the same liquid (Ross \& Miles, 1941). Therefore, it is interpretable as a lab scale counterpart of the filling process. In fact, the morphology and bubble size distributions of the generated foams in filling experiments are very similar to those of foams which can be created by Ross-Miles test (Rosen \& Solash, 1969). Based on those results, future investigations should address, if Ross-Miles test is applicable to predict foam generation in bottling processes a priori. That would be helpful for producers of liquid foods and beverages, since contaminations of bottling plants due to excessive foaming could be preventable.

The determination of bubble characteristics in foams and liquids in industry-like processes enabled to correlate the measured properties to process characteristics. As an example of application, correlations in terms of the Capillary number were determined. In industrial processes, this information is not easy to obtain due to nonideal or perturbed conditions for image acquisition, so that image processing with CNNs seems to be beneficial for this task. Generally, the investigated plunging jet configuration is not only important for food processing. Similar configurations can be found in other industries, for instance in bioreactors, nuclear reactors, wastewater treatment facilities, gas-liquid separators or they are encountered in nature (Chanson et al., 2006; Duarte et al., 
2016; Guyot et al., 2019; Pagliara \& Palermo, 2017; Roy et al., 2013; Schmidtke \& Lucas, 2009; Somasundaran, 1975). Although some data of BSDs are reported (Atkinson et al., 2003; Boyd \& Varley, 2004; Evans et al., 1992), little information is available on how process and material characteristics affect the BSD in a plunging jet configuration. To the authors' knowledge, no correlations of the BSD of entrained gas bubbles with process characteristics are available so that their measurement in industrial processes and the correlation to process conditions seem to be a beneficial field of research.

Since the employed CNN turned out to be a robust tool for the segmentation of foam images, it is also usable for further research on the impact of process conditions on the morphology and BSD in foams. Therefore, different goals seem to be possible. With specific regard to bottling, it is reasonable to apply the developed methods to investigate foaming during the bottling of carbonated beverages. In this case, the release of dissolved gases must be considered as an additional mechanism of gas bubble formation besides gas entrainment by the filling jet (Salerno et al., 2017). It is known that the gas release from beverages depends on several parameters, such as the degree of supersaturation, surface tension, temperature and pressure (Fischer, 2001). However, the relation of process parameters, such as the filling speed to the local hydrodynamic pressure, to the rate of nucleation and to gas release is still open to further research. It can be expected from the reported literature that both mechanisms of bubble formation lead to gas bubbles of very different sizes, which will also affect the rate of foam formation and foam stability. The knowledge about the impacts of a process on the morphology of disturbing foams, which is a dominant feature of their stability, might help to better design industrial processes. In such a way, foaming is reducible or ideally preventable, which would be also beneficial for other processes than bottling. The literature does not contain much information about this topic, although foaming is a serious problem under industrial conditions and, indeed, a huge demand for further research exists. On the other hand, foams may be desirable if they are part of a product, which is the case for many foods. Relating foam characteristics to process effects might enable to design foams with special characteristics and therefore to improve the functional and quality properties of food products.

\section{Conclusion}

The photo-optical data acquisition in real geometries of industrial processes often results in complex images due to the superposition of optical phenomena in the considered multiphase systems. Although obviously identifiable by the human eye, classic image analysis on those data is failure-prone due to changes in lighting conditions. Therefore, the measurements usually take place in bypasses with narrow measurement chambers to keep the lighting conditions steady and to allow an object segmentation. Since, especially in delicate disperse systems like foam, that way of data acquisition strongly influences the measured variable itself, the measurement technique should adapt to the system and not vice versa, i.e., being minimally invasive and applicable to real geometries. The implementation of CNNs approaches this difficult task by means of software and shows impressive results. Although one main advantage of both presented CNNs is the relatively small amount of manually labeled training data, which is required as segmentation target, still, the parametrization and network training turns out to be the most time-consuming step.

In this study, the modified CNNs are employed for an industrial application using the example of a juice bottling process with air bubbles being entrained by a plunging jet. The measurement results of the consequent foam reveal various morphology parameters and allow an interpretation of the influence of system specific variables, as well as operating conditions of the filling process. This is exemplarily shown by approximating the bubble sizes with distribution functions, classifying the foam into the wet disordered regime, interpreting inertial, viscous and interfacial forces by means of dimensionless numbers and correlating with process parameters of the test rig like filling speed. Henceforth, the developed method is usable to generate additional data points in order to underpin or revise the proposed correlations and interpretations. 


\section{Acknowledgments}

The IGF project of the FEI (AiF $19711 \mathrm{~N}$ ) within the program for promoting the "Industrial Collective Research” (IGF) and the ZIM cooperation project (SPI, ZF4184301CR5 \& ZF4184501CR5) within the „Central Innovation Programme for SMEs" (ZIM) are supported via AiF by the German Federal Ministry for Economic Affairs and Energy (BMWi) on the basis of a decision by the German Bundestag. The authors thank for the financial support.

\section{Declaration of Interest}

The authors Robert P. Panckow, Michael Muthig and Mirco Wegener are employed at SOPAT GmbH that is supported in the ZIM cooperation project via AiF. SOPAT GmbH is producer and seller of hardware and software for particle size and shape measurement in multiphase systems. For the rest of the authors, there are no interests to declare.

\section{References}

Atkinson, B. W., Jameson, G. J., Nguyen, A. V., Evans, G. M., \& Machniewski, P. M. (2003). Bubble Breakup and Coalescence in a Plunging Liquid Jet Bubble Column. The Canadian Journal of Chemical Engineering, 81(3-4), 519-527. https://doi.org/10.1002/cjce.5450810325

Bamforth, C. W. (2004). The Relative Significance of Physics and Chemistry for Beer Foam Excellence: Theory and Practice. Journal of the Institute of Brewing, 110(4), 259-266. https://doi.org/10.1002/j.2050-0416.2004.tb00620.x

Barik, T. K., \& Roy, A. (2009). Statistical distribution of bubble size in wet foam. Chemical Engineering Science, 64(9), $2039-2043$. https://doi.org/10.1016/j.ces.2009.01.039

Bertola, N., Wang, H., \& Chanson, H. (2018). Air Bubble Entrainment, Breakup, and Interplay in Vertical Plunging Jets. Journal of Fluids Engineering, 140(9). https://doi.org/10.1115/1.4039715

Blüml, S., \& Fischer, S. (2004). Manual of Filling Technology - The Theory and Practice of Filling Liquid Products (First Edit). Behrs.

Borcherding, K., Lorenzen, P. C., Hoffmann, W., \& Schrader, K. (2008). Effect of foaming temperature and varying time/temperatureconditions of pre-heating on the foaming properties of skimmed milk. International Dairy Journal, 18(4), $349-358$. https://doi.org/10.1016/j.idairyj.2007.11.016

Bottou, L. (2012). Stochastic gradient descent tricks. Lecture Notes in Computer Science (Including Subseries Lecture Notes in Artificial Intelligence and Lecture Notes in Bioinformatics), 7700 LECTU(1), 421-436. https://doi.org/10.1007/978-3-642-35289-8-25

Boyd, J. W. R., \& Varley, J. (2004). Acoustic emission measurement of low velocity plunging jets to monitor bubble size. Chemical Engineering Journal, 97(1), 11-25. https://doi.org/10.1016/S1385-8947(03)00115-3

Cantat, I., Cohen-Addad, S., Elias, F., Graner, F., Höhler, R., Pitois, O., Rouyer, F., Saint-Jalmes, A., \& Flatman, R. (2013). Foams (S. Cox (ed.)). Oxford University Press. https://doi.org/10.1093/acprof:oso/9780199662890.001.0001

Chandran Suja, V., Kar, A., Cates, W., Remmert, S. M., Savage, P. D., \& Fuller, G. G. (2018). Evaporation-induced foam stabilization in lubricating oils. Proceedings of the National Academy of Sciences, 115(31), $7919-7924$. https://doi.org/10.1073/pnas.1805645115

Chanson, H., Aoki, S., \& Hoque, A. (2006). Bubble Entrainment and Dispersion in Plunging Jet Flows: Freshwater vs. Seawater. Journal of Coastal Research, 223(223), 664-677. https://doi.org/10.2112/03-0112.1

Cheng, H. C., \& Lemlich, R. (1983). Errors in the measurement of bubble size distribution in foam. Industrial \& Engineering Chemistry Fundamentals, 22(1), 105-109. https://doi.org/10.1021/i100009a018

Colombet, D., Legendre, D., Risso, F., Cockx, A., \& Guiraud, P. (2015). Dynamics and mass transfer of rising bubbles in a homogenous swarm at large gas volume fraction. Journal of Fluid Mechanics, 763, 254-285. https://doi.org/10.1017/jfm.2014.672

Delgado, A., Peukert, W., Miller, R., Willenbacher, N., Kulozik, U., Hanke, R., Hinrichs, J., Schieberle, P., Becker, T., \& Rüde, U. (2014). Proteinschäume in der Lebensmittelproduktion: Mechanismenaufklärung, Modellierung und Simulation (V. Häusser \& D. Kinkel (eds.)). Forschungskreis der Ernährungsindustrie e.V. (FEI). https://www.feibonn.de/mediathek/print/sonderpublikationen/abschlusspublikation-proteinschaeume

Den Engelsen, C. W., Isarin, J. C., Gooijer, H., Warmoeskerken, M. M. C. G., \& Wassink, J. G. (2002). Bubble Size Distribution of Foam. Autex Research Journal, 2(1).

Dold, S., Lindinger, C., Kolodziejczyk, E., Pollien, P., Ali, S., Germain, J. C., Perin, S. G., Pineau, N., Folmer, B., Engel, K.-H., Barron, D., \& Hartmann, C. (2011). Influence of Foam Structure on the Release Kinetics of Volatiles from Espresso Coffee Prior to Consumption. Journal of Agricultural and Food Chemistry, 59(20), 11196-11203. https://doi.org/10.1021/jf201758h

Drenckhan, W., \& Hutzler, S. (2015). Structure and energy of liquid foams. Advances in Colloid and Interface Science, 224, 1-16. https://doi.org/10.1016/j.cis.2015.05.004

Drenckhan, W., \& Saint-Jalmes, A. (2015). The science of foaming. Advances in Colloid and Interface Science, 222, $228-259$. https://doi.org/10.1016/j.cis.2015.04.001

Duarte, R., Pinheiro, A., \& Schleiss, A. J. (2016). An Enhanced Physically Based Scour Model for Considering Jet Air Entrainment. Engineering, 2(3), 294-301. https://doi.org/10.1016/J.ENG.2016.03.003

Evans, G. M., Jameson, G. J., \& Atkinson, B. W. (1992). Prediction of the bubble size generated by a plunging liquid jet bubble column. 
Chemical Engineering Science, 47(13-14), 3265-3272. https://doi.org/10.1016/0009-2509(92)85034-9

Feitosa, K., Halt, O. L., Kamien, R. D., \& Durian, D. J. (2006). Bubble kinetics in a steady-state column of aqueous foam. Europhysics Letters (EPL), 76(4), 683-689. https://doi.org/10.1209/epl/i2006-10304-5

Fischer, S. (2001). Blasenbildung von in Flüssigkeiten gelösten Gasen. Technische Universität München.

Germain, J. C., \& Aguilera, J. M. (2012). Identifying industrial food foam structures by 2D surface image analysis and pattern recognition. Journal of Food Engineering, 111(2), 440-448. https://doi.org/10.1016/j.jfoodeng.2012.01.018

Girshick, R. (2015). Fast R-CNN. 2015 IEEE International Conference on Computer Vision (ICCV), 2015 Inter, 1440-1448. https://doi.org/10.1109/ICCV.2015.169

Girshick, R., Donahue, J., Darrell, T., \& Malik, J. (2014). Rich Feature Hierarchies for Accurate Object Detection and Semantic Segmentation. 2014 IEEE Conference on Computer Vision and Pattern Recognition, 580-587. https://doi.org/10.1109/CVPR.2014.81

Guyot, G., Cartellier, A., \& Matas, J.-P. (2019). Depth of penetration of bubbles entrained by an oscillated plunging water jet. Chemical Engineering Science: X, 2, 100017. https://doi.org/10.1016/j.cesx.2019.100017

Hanbury, A. (2003). Mathematical Morphology Applied to Circular Data (pp. 123-IN2). https://doi.org/10.1016/S1076-5670(03)800642

Ionescu-Tirgoviste, C., Gagniuc, P. A., Gubceac, E., Mardare, L., Popescu, I., Dima, S., \& Militaru, M. (2015). A 3D map of the islet routes throughout the healthy human pancreas. Scientific Reports, 5(1), 14634. https://doi.org/10.1038/srep14634

Jia, Y., Shelhamer, E., Donahue, J., Karayev, S., Long, J., Girshick, R., Guadarrama, S., \& Darrell, T. (2014). Caffe: Convolutional Architecture for Fast Feature Embedding. Proceedings of the ACM International Conference on Multimedia - MM '14, 675-678. https://doi.org/10.1145/2647868.2654889

Kiger, K. T., \& Duncan, J. H. (2012). Air-Entrainment Mechanisms in Plunging Jets and Breaking Waves. Annual Review of Fluid Mechanics, 44(1), 563-596. https://doi.org/10.1146/annurev-fluid-122109-160724

Kingma, D. P., \& Ba, J. (2017). Adam: A Method for Stochastic Optimization. 1-15. http://arxiv.org/abs/1412.6980v9

Maßß, S., Rojahn, J., Hänsch, R., \& Kraume, M. (2012). Automated drop detection using image analysis for online particle size monitoring in multiphase systems. Computers \& Chemical Engineering, 45, 27-37. https://doi.org/10.1016/j.compchemeng.2012.05.014

Mack, S., Hussein, M. A., \& Becker, T. (2011). Modeling flavor development in cereal based foams under thermal treatment. Procedia Food Science, 1, 1223-1230. https://doi.org/10.1016/j.profoo.2011.09.182

Magrabi, S. A., Dlugogorski, B. Z., \& Jameson, G. J. (1999). Bubble size distribution and coarsening of aqueous foams. Chemical Engineering Science, 54(18), 4007-4022. https://doi.org/10.1016/S0009-2509(99)00098-6

Minor, M., Vingerhoeds, M. H., Zoet, F. D., de Wijk, R., \& van Aken, G. A. (2009). Preparation and sensory perception of fat-free foams - effect of matrix properties and level of aeration. International Journal of Food Science \& Technology, 44(4), 735-747. https://doi.org/10.1111/j.1365-2621.2008.01887.x

Pagliara, S., \& Palermo, M. (2017). Scour process caused by multiple subvertical non-crossing jets. Water Science and Engineering, 10(1), 17-24. https://doi.org/10.1016/j.wse.2017.03.010

Panckow, R. P., Reinecke, L., Cuellar, M. C., \& Maa, S. (2017). Photo-Optical In-Situ Measurement of Drop Size Distributions: Applications in Research and Industry. Oil and Gas Science and Technology, 72(3). https://doi.org/10.2516/ogst/2017009

Politova, N., Tcholakova, S., Valkova, Z., Golemanov, K., \& Denkov, N. D. (2018). Self-regulation of foam volume and bubble size during foaming via shear mixing. Colloids and Surfaces A: Physicochemical and Engineering Aspects, 539(November 2017), 1828. https://doi.org/10.1016/j.colsurfa.2017.12.006

Rami-shojaei, S., Vachier, C., \& Schmitt, C. (2009). Automatic analysis of 2D foam sequences: Application to the characterization of aqueous proteins foams stability. Image and Vision Computing, 27(6), 609-622. https://doi.org/10.1016/j.imavis.2008.10.004

Reglitz, K. (2016). Influence of composition and structure on the release of aroma compounds from foamed milk model systems. Technische Universität München.

Ren, S., He, K., Girshick, R., \& Sun, J. (2017). Faster R-CNN: Towards Real-Time Object Detection with Region Proposal Networks. IEEE Transactions on Pattern Analysis and Machine Intelligence, 39(6), 1137-1149. https://doi.org/10.1109/TPAMI.2016.2577031

Roberts, S. A., \& Rao, R. R. (2011). Numerical simulations of mounding and submerging flows of shear-thinning jets impinging in a container. Journal of Non-Newtonian Fluid Mechanics, 166(19-20), 1100-1115. https://doi.org/10.1016/j.jnnfm.2011.06.006

Ronneberger, O., Fischer, P., \& Brox, T. (2015). U-net: Convolutional networks for biomedical image segmentation. Lecture Notes in Computer Science (Including Subseries Lecture Notes in Artificial Intelligence and Lecture Notes in Bioinformatics), 9351,234 241. https://doi.org/10.1007/978-3-319-24574-4_28

Rosen, M. J., \& Solash, J. (1969). Factors affecting initial foam height in the Ross-Miles foam test. Journal of the American Oil Chemists Society, 46(8), 399-402. https://doi.org/10.1007/BF02545623

Ross, J., \& Miles, G. D. (1941). An apparatus for comparison of foaming properties of soaps and detergents. Oil \& Soap, 18(5), 99-102. https://doi.org/10.1007/BF02545418

Roy, A. K., Maiti, B., \& Das, P. K. (2013). Visualisation of Air Entrainment by a Plunging Jet. Procedia Engineering, 56, $468-473$. https://doi.org/10.1016/j.proeng.2013.03.148

Safouane, M., Saint-Jalmes, A., Bergeron, V., \& Langevin, D. (2006). Viscosity effects in foam drainage: Newtonian and non-newtonian foaming fluids. The European Physical Journal E, 19(2), 195-202. https://doi.org/10.1140/epje/e2006-00025-4

Saint-Jalmes, A. (2006). Physical chemistry in foam drainage and coarsening. Soft Matter, 2(10), 836. https://doi.org/10.1039/b606780h

Salerno, E., Levoni, P., Barozzi, G. S., \& Malfatto, A. (2017). Foam evolution in a processed liquid solution. Journal of Physics: Conference Series, 796, 012042. https://doi.org/10.1088/1742-6596/796/1/012042

Schäfer, J., Schmitt, P., Hlawitschka, M. W., \& Bart, H. (2019). Measuring Particle Size Distributions in Multiphase Flows Using a Convolutional Neural Network. Chemie Ingenieur Technik, 91(11), 1688-1695. https://doi.org/10.1002/cite.201900099

Schmidtke, M., \& Lucas, D. (2009). CFD Approaches for Modelling Bubble Entrainment by an Impinging Jet. Science and Technology of Nuclear Installations, 2009, 1-12. https://doi.org/10.1155/2009/148436 
Schramm, L. L., \& Wassmuth, F. (1994). Foams: Basic Principles. In PALAIOS (Vol. 16, Issue 6, pp. 3-45). https://doi.org/10.1021/ba1994-0242.ch001

Senge, B., Blochwitz, R., \& Bentlin, S. (2004). Rheologische Stoffkennwerte richtig bestimmen. DMW - Die Milchwirtschaft, 7, 256260. https://www.lmr.tu-berlin.de/fileadmin/fg78/Download/stoffkennwerte.pdf

Soleymani, M., Kamali, M. R., \& Saeedabadian, Y. (2013). Experimental Investigation of Physical and Chemical Properties of Drilling Foam and Increasing its Stability. Iranian Journal of Chemistry and Chemical Engineering, 32(3), $127-132$. http://www.ijcce.ac.ir/article 5838.html

Somasundaran, P. (1975). Separation Using Foaming Techniques. Separation Science, 10(1), 93-109. https://doi.org/10.1080/00372367508057071

Stanley, D. W., Goff, H. D., \& Smith, A. K. (1996). Texture-structure relationships in foamed dairy emulsions. Food Research International, 29(1), 1-13. https://doi.org/10.1016/0963-9969(95)00063-1

van Hecke, M. (2010). Jamming of soft particles: geometry, mechanics, scaling and isostaticity. Journal of Physics: Condensed Matter, 22(3), 033101. https://doi.org/10.1088/0953-8984/22/3/033101

Vera, M. U., Saint-Jalmes, A., \& Durian, D. J. (2001). Scattering optics of foam. Applied Optics, 40(24), 4210. https://doi.org/10.1364/AO.40.004210

Ziegenhein, T., Zalucky, J., Rzehak, R., \& Lucas, D. (2016). On the hydrodynamics of airlift reactors, Part I: Experiments. Chemical Engineering Science, 150, 54-65. https://doi.org/10.1016/j.ces.2016.04.039 